\title{
Effect of acupoint catgut embedding therapy combined with Chinese medicine for nourishing the kidneys and promoting blood circulation and improving blood glucose and lipid levels as well as the pregnancy rate in obese PCOS patients with infertility
}

\author{
WENMIN QIN, KAI ZHAO and HAIYAN YANG
}

\begin{abstract}
Department of Gynaecology, Xuzhou Hospital of Traditional Chinese Medicine, Xuzhou, Jiangsu 221003, P.R. China
\end{abstract}
Received June 28, 2016; Accepted September 16, 2016

DOI: $10.3892 /$ etm.2016.3715

\begin{abstract}
In this study, we explored the effect of acupoint catgut embedding combined with Chinese medicine for nourishing kidney and promoting blood circulation as well as improving the glucose and lipid levels and pregnancy rate in obese polycystic ovary syndrome (PCOS) patients with infertility. A total of 62 obese patients with PCOS were randomly divided into three groups, 21 of whom were treated with acupoint catgut embedding combined with Chinese medicine, another 21 with catgut embedding therapy, and 20 only with Chinese medicine. We compared the improvement of clinical symptoms and the glucose and lipid levels in the three groups three months after surgery. We found that the effective rate of obesity treatment, body mass index and waist-to-hip ratio of acupuncturedrug group and catgut embedding group were significantly lower than those of the Chinese medicine group $(\mathrm{P}<0.05)$. A comparison between the acupuncture-drug group and catgut embedding group was not statistically significant $(\mathrm{P}>0.05)$. The triglyceride (TG) and high-density lipoprotein cholesterol (HDL) levels of the three groups were significantly decreased and increased, respectively, following treatment, with the total cholesterol (TC) level being decreased significantly only
\end{abstract}

Correspondence to: Dr Wenmin Qin, Department of Gynaecology, Xuzhou Hospital of Traditional Chinese Medicine, 169 Zhongshan South Road, Xuzhou, Jiangsu 221003, P.R. China

E-mail: fgwahoopwb35@163.com

Abbreviations: PCOS, polycystic ovary syndrome; FPG, fasting plasma glucose; FNS, fast insulins; BMI, body mass index; HCG, human chorionic gonadotropin; WHR, waist-to-hip ratio; TC, total cholesterol; TG, triglyceride; HDL, high-density lipoprotein cholesterol; LDL, low-density lipoprotein cholesterol; LUF, luteinized unruptured follicle; OHSS, ovarian hyper-stimulation syndrome

Key words: polycystic ovarian syndrome, acupoin catgut embedding, Chinese medicine of nourishing kidney and promoting blood circulation, spectrum of glucose and lipid, pregnancy rate in the acupuncture-drug group $(\mathrm{P}<0.05)$, and the LDL level showing no significant difference $(\mathrm{P}>0.05)$. The fast insulins, HOMA-IR, TG, TC levels and periodic cancellation rate of the acupuncture-drug group was significantly reduced compared to those of the catgut embedding group and Chinese medicine group after treatment, whereas the HDL levels, periodic ovulation rate and clinical pregnancy rate were higher $(\mathrm{P}<0.05)$. However, a comparison between Chinese medicine group and catgut embedding group was not statistically significant $(\mathrm{P}>0.05)$. The incidence rate of luteinized unruptured follicle and ovarian hyper-stimulation syndrome of the three groups showed no statistical difference $(\mathrm{P}>0.05)$. In conclusion, the study has shown that acupoint catgut embedding therapy combined with Chinese medicine for nourishing the kidneys and promoting blood circulation and improving blood glucose and lipid levels as well as the pregnancy rate in obese PCOS patients with infertility is useful.

\section{Introduction}

Polycystic ovary syndrome (PCOS) is a comprehensive syndrome with the characteristics of chronic anovulation and polycystic ovary, and with low menstruation or amenorrhea, obesity, crinosity and infertility as main clinical features $(1,2)$. It not only involves the genital system, but also directly influences the fertility function of women, and is a type of metabolic syndrome with hyperinsulinemia, hyperlipidemia, diabetes and frequent cardiovascular diseases. It involves multi-disciplinary problems in treatment, e.g., menoxenia, fertility issues, intimal hyperplasia and long-term metabolic syndrome. Therefore, with the deepening of research, PCOS has gone beyond the range of gynecology. Its pathomechanism involves multiple systems including heredity, internal secretion, immunity and metabolism, and its physical and mental damage to patients have raised the attention, which has become the difficulty and focus of gynecological field $(3,4)$. The characteristics of traditional Chinese medicines including holistic concept, treatment based on syndrome differentiation, multi-system and multiple target point are unique advantages and consitute great potential in the treatment of PCOS $(5,6)$. 
Table I. Comparison of age, medical history, BMI of the three groups of PCOS patients before treatment (mean \pm SD).

\begin{tabular}{lccccc}
\hline Groups & Cases & Age (years) & Medical history (years) & WHR & BMI \\
\hline Acupuncture-drug & 21 & $28.33 \pm 5.12$ & $6.26 \pm 1.66$ & $0.89 \pm 0.09$ & $27.44 \pm 2.35$ \\
Catgut embedding & 21 & $29.02 \pm 4.87$ & $6.18 \pm 1.85$ & $0.88 \pm 0.09$ & $27.67 \pm 1.85$ \\
Chinese medicine & 20 & $28.77 \pm 4.77$ & $6.82 \pm 1.77$ & $0.89 \pm 0.07$ & $27.06 \pm 2.01$ \\
\hline
\end{tabular}

BMI, body mass index; PCOS, polycystic ovary syndrome; WHR, weight-to-hip ratio.

Table II. Comparison of glucose metabolism index of the three groups of PCOS patients before treatment (mean \pm SD).

\begin{tabular}{lcccc}
\hline Groups & Cases & FPG (mmol/l) & FNS (mmol/l) & HOMA-IR \\
\hline Acupuncture-drug & 21 & $4.23 \pm 0.81$ & $17.12 \pm 6.24$ & $3.22 \pm 1.59$ \\
Catgut embedding & 21 & $4.39 \pm 1.02$ & $18.26 \pm 7.25$ & $3.58 \pm 1.69$ \\
Chinese medicine & 20 & $4.51 \pm 1.06$ & $19.33 \pm 6.77$ & $3.87 \pm 1.74$ \\
\hline
\end{tabular}

PCOS, polycystic ovary syndrome; FPG, fasting plasma glucose; FNS, fast insulins.

Table III. Comparison of lipid profile of the three groups of PCOS patients before treatment ( $\mathrm{mmol} / \mathrm{l}$, mean $\pm \mathrm{SD}$ ).

\begin{tabular}{lccccc}
\hline Groups & Cases & TC & TG & LDL & HDL \\
\hline Acupuncture-drug & 21 & $4.67 \pm 0.59$ & $2.02 \pm 0.85$ & $2.96 \pm 0.51$ & $1.27 \pm 0.42$ \\
Catgut embedding & 21 & $4.48 \pm 0.64$ & $2.19 \pm 0.93$ & $3.11 \pm 0.57$ & $1.18 \pm 0.36$ \\
Chinese medicine & 20 & $4.65 \pm 0.77$ & $2.22 \pm 0.79$ & $3.08 \pm 0.55$ & $1.15 \pm 0.41$ \\
\hline
\end{tabular}

PCOS, polycystic ovary syndrome; TC, total cholesterol; TG, triglyceride; LDL, low-density lipoprotein cholesterol; HDL, high-density lipoprotein cholesterol.

In the present study, we examined the impact of acupoint catgut embedding therapy combined with Chinese medicine for nourishing the kidneys and promoting blood circulation and improving blood glucose and lipid levels as well as the pregnancy rate in obese PCOS patients with infertility.

\section{Materials and methods}

General materials. All the cases were from the Department of Gynecology at the Xuzhou Hospital of Traditional Chinese Medicine (Jiangsu, China) between January, 2012 and December, 2014. A total of 62 cases with endomorphy-type PCOS that conforms to diagnostic criteria were selected and randomly divided into three groups. The acupuncture-drug group comprised 21 cases; the Chinese medicine group, 20 cases; and the catgut embedding group, 21 cases. Before treatment, the age, medical history and weight of the three groups were compared (Table I).

Prior to treatment, fasting plasma glucose (FPG), fast insulins (FNS), HOMA - IR = FPG x FNS/22.5, total cholesterol (TC), triglyceride (TG), low-density lipoprotein cholesterol (LDL), and the high-density lipoprotein cholesterol (HDL) of the three groups were compared, and the difference was not statistically significant $(P>0.05)$ (Tables II and III).
Diagnostic criteria of PCOS. The criteria were formulated at the 2003 Rotterdam POC discussion jointly launched by the European Society of Human Reproduction and Embryology (ESHRE) and the American Society for Reproductive Medicine (ASRM) (1): a) Occasional ovulation and (or) no ovulation; b) clinical and (or) biochemical indexes suggest the existence of hyperandrogenism, and possible pathogenic factors such as congenital adrenal hyperplasia, androgen tumor secretion and Cushing's syndrome were all excluded. [The clinical features of hyperandrogenism include acne, crinosity and baldness, and biochemical indexes should refer to the standard of $\mathrm{T} \geq 2.2 \mathrm{nmol} / \mathrm{l}$ proposed by $\mathrm{Li} \mathrm{(2)];} \mathrm{and}$ c) bilateral polycystic changes of the ovaries: The volume of bilateral ovaries increased, and there were $>10$ small follicles on each side, diameters ranging from 2 to $8 \mathrm{~mm}$.

If two or three aforementioned criteria of i)-iii) were met, the patients were diagnosed as PCOS.

Diagnostic criteria of obesity. The diagnosis of obesity referred to Asian adult body mass index (BMI) criteria defined and proposed by the World Health Organization (WHO) Western Pacific region obesity working group in 2000, BMI $=$ weight $(\mathrm{kg}) /$ height $(\mathrm{m})$. BMI ranging from 23.0 to 24.9 was regarded as pre-obesity, 25.0-29.9 was regarded as level I obesity, $\geq 30.0$ was regarded as level II obesity. 
Abdominal obesity diagnostic criteria refers to the definition of Asian people abdominal obesity proposed by WHO in 1998: Waistline, men $>90 \mathrm{~cm}$ or women $>80 \mathrm{~cm}$. The measurement of height was done barefoot with accuracy to $1 \mathrm{~cm}$; weight was measured by electronic scale; cross-section diameter was measured by flexible rule standing with accuracy to $1 \mathrm{~cm}$, abdominal girth was obtained by the cross-section diameter of the mid-point between low edge of the 12th rib and pre-iliac spines, and hipline was obtained by the cross-section diameter of the most prominent level of hip. BMI and waist-to-hip ratio (WHR) were calculated according to this, and professional staff were responsible for all indexes above.

Traditional Chinese medical syndrome differentiation standards of renal deficiency and blood stasis. According to the relevant contents of the Clinical Research Guidance Principle of Treating Irregular Menstruation by New Traditional Chinese Medicines (5), and the 7th edition of Gynecology of Chinese Medicine: a) Menstrual cycle postponed, menstrual blood volume was low, the color was dull-red and has coagula, had abdominal pain, or gradual amenorrhea, or no pregnancy long after marriage; b) waist and knee were aching and limp, fatigue, abdominal was aching as in acupuncture, and urination was frequent during the night; c) the tongue was dull, or had petechia at one side, the fur was white, and pulse was deep. For aforementioned conditions, a) and c) were required, and if 1 or 2 options in b) were met, PCOS was diagnosed.

Inclusion criteria. Patient ages ranged from 20 to 42 years, and simultaneously conformed to the diagnostic criteria, obesity diagnostic criteria and traditional Chinese medical syndrome differentiation standards of PCOS. Written informed consent was provided.

Exclusion criteria. i) Patients with other endocrine diseases or in combination with serious primary diseases in heart, liver, kidney, patients with mental disorders; ii) hormone drugs used in recent three months; iii) patients allergic to traditional Chinese medicines or unable to bear thread implanting treatments; and iv) patients not conforming to inclusion criteria or not using drugs and receiving treatments according to the regulations, factors that influence curative effects or safety such as curative effects could not be judged as statistics were incomplete.

Acupuncture-drug group. Acupoint catgut embedding in combination with traditional Chinese medicines were used for treatment: i) Acupoint catgut embedding, the acupoints of Zhongwan, Shenyu, Zhongji, Guanyuan, Xuehai, Sanyinjiao, Fenglong and Zusanli were selected. The acupuncture apparatus was disposable and inlcuded no. 7 needle, $0.3 \times 50 \mathrm{~mm}$ stainless steel flat needle was used for stylet, and 3-0 medical catgut was cut into $1 \mathrm{~cm}$ line segments to soak into $75 \%$ alcohol for preparation. While aiming at the selected acupoints and rapidly transdermal, the needle was inserted slowly. Subsequently, it was pushed in stylet while withdrawing needle tubing, to leave catgut in acupoints (catgut is left between subcutaneous tissue and muscle, and could not be left in subcutaneous tissue. After withdrawing the needle, sterilized cotton ball was used to press the pinhole for a while to prevent hemorrhage, medical proof fabric was used later for the external application of pinholes, and it was required that the catgut hole should not touch water. Thread implanting treatment involved avoiding menstrual period, treatment every 7-10 days, and continued for 3 months. ii) Taking kidney-supplementing and blood-quickening decoction, the medicines included: Angelica sinensis $15 \mathrm{~g}$, Ligusticum wallichii $10 \mathrm{~g}$, Radix Paeoniae Alba $15 \mathrm{~g}$, Rehmannia glutinosa $15 \mathrm{~g}$, Herba epimedii $15 \mathrm{~g}$, Eucommia ulmoides $15 \mathrm{~g}$, Fructus Corni $15 \mathrm{~g}$, Rhizoma Cyperi 10 g, Radix Paeoniae Rubra 15 g, Cortex Moutan 15 g, Spina Gleditsiae 10 g, Rhizoma Pinellinae Praeparata 10 g, Pericarpium Citri Reticulatae 6 g, one dose a day, decocted by water and not taken during the menstrual period.

Chinese medicine group. The group was treated with the decoction for nourishing the kidney and promoting blood circulation, and the remaining procedure was identical to that of the acupuncture-drug group.

Catgut embedding group. Similarly, following treatment with acupoint catgut embedding, the remaining procedure was identical to that of the acupuncture medicine group.

Weight loss and glucolipid metabolism indexes in the three groups after the treatment were observed, while at the same time, starting from the 5 th day of the menstrual cycle, Clomiphene was continually taken orally (CC; Codal Synto Ltd., Shanghai, China) 50-100 mg/day for 5 days, and B ultrasound detection showed that there was the development of non-advantageous follicle, and HMG (Lizhu Pharmaceutical Trading Co., Ltd., Zhuhai, China) was used according to the supplier conditions. The diameter of follicle was $>18 \mathrm{~mm}$, human chorionic gonadotropin (HCG) was injected to the muscle (Lizhu Pharmaceutical Trading Co., Ltd.), and Duphaston was orally taken after ovulation to carry out luteal support, basic body temperature was self-measured, and HCG in urine was examined 14-16 days after ovulation, if $\mathrm{HCG}(+)$, clinical pregnancy was confirmed by B ultrasound monitoring 35-38 days after ovulation.

If follicles whose diameters were $>15 \mathrm{~mm}$ were $>3, \mathrm{HCG}$ muscle injection was stopped, and the cycle was cancelled to prevent the occurrence of ovarian hyper-stimulation syndrome (OHSS) and multiple pregnancy.

Improvement of clinical symptoms. The improvements of weight, waistline and pregnancy of the three groups were compared before and after treatment, and BMI, WHR and clinical pregnancy rate of patients before and after treatment was calculated.

Determination of glucose and lipid index. After treatment, on the morning of the second day or the third day after menstruation was over, fasting venous blood of $2 \mathrm{ml}$ was taken and examined for FPG, FNS, TC, TG, HDL and LDL.

Curative effects evaluation criteria. Clinical cure of obesity curative criteria included reduction in weight, which reached standard body weight or overweight range. Scoring was as follows: Excellent, decline in weight of $>5 \mathrm{~kg}$ or decline in fat of $>5 \%$; effective, weight decline of $>3 \mathrm{~kg}$ or fat decline of $5 \%$; and non-effective, decline in weight $<3 \mathrm{~kg}$ or fat decline 
Table IV. Comparison of the result of the three groups of obese PCOS patients after treatment.

\begin{tabular}{lcccccc}
\hline Groups & Cases & Clinical cure & Excellent & Valid & Invalid & Effective rate (100\%) \\
\hline Acupuncture-drug & 19 & 4 & 7 & 5 & 3 & 84.21 \\
Catgut embedding & 20 & 3 & 7 & 6 & 4 & $80.00^{\text {a }}$ \\
Chinese medicine & 18 & 1 & 3 & 5 & 9 & $50.00^{\mathrm{b}}$ \\
\hline
\end{tabular}

Compared with acupuncture-drug group, ${ }^{\mathrm{a}} \mathrm{P}>0.05$; compared with acupuncture-drug group and catgut embedding group, ${ }^{\mathrm{b}} \mathrm{P}<0.05$. $\mathrm{PCOS}$, polycystic ovary syndrome.

Table V. The comparison of BMI,WHR and glucose metabolism index of the three groups of obese PCOS patients before and after treatment.

\begin{tabular}{lccccc}
\hline Groups/time & Cases & BMI $\left(\mathrm{kg} / \mathrm{m}^{2}\right)$ & WHR & FPG $(\mathrm{mmol} / \mathrm{l})$ & FNS (mmol/l) \\
\hline $\begin{array}{l}\text { Acupuncture } \\
\text { Before treatment }\end{array}$ & 21 & $27.44 \pm 2.35$ & $0.89 \pm 0.09$ & $4.23 \pm 0.81$ & $17.12 \pm 6.24$ \\
After treatment & 19 & $23.94 \pm 1.89$ & $0.85 \pm 0.07$ & $3.88 \pm 0.62$ & $10.39 \pm 4.88$ \\
Catgut embedding & & & & & \\
Before treatment & 21 & $27.67 \pm 1.85$ & $0.88 \pm 0.09$ & $4.39 \pm 1.02$ & $18.26 \pm 7.25$ \\
After treatment & 20 & $24.14 \pm 2.02^{\mathrm{a}}$ & $0.86 \pm 0.06^{\mathrm{a}}$ & $3.96 \pm 0.98$ & 1.59 \\
Chinese medicine & & & & & $3.58 \pm 1.69$ \\
Before treatment & 20 & $27.06 \pm 2.01$ & $0.89 \pm 0.07$ & $4.51 \pm 1.06$ & $19.33 \pm 6.77$ \\
After treatment & 18 & $25.36 \pm 2.41^{\mathrm{b}}$ & $0.88 \pm 0.08^{\mathrm{b}}$ & $3.90 \pm 1.07$ & $12.43 \pm 4.09^{\mathrm{b}}$ \\
\hline
\end{tabular}

Compared with the before treatment, $\mathrm{P}<0.05$; compared with Chinese medicine group, ${ }^{\mathrm{a}} \mathrm{P}<0.05$; compared with acupuncture-drug group, ${ }^{\mathrm{b}} \mathrm{P}<0.05$. BMI, body mass index; WHR, waist-to-hip ratio; PCOS, polycystic ovary syndrome; FPG, fasting plasma glucose; FNS, fast insulins.

$<5 \%$ (7). Clinical pregnancy conditions were counted after the induction of ovulation.

Statistical analysis. SPSS 13.0 statistical analysis software (Chicago, IL, USA) was used. The measured data were expressed by mean \pm standard deviation (SD). One-way analysis of variance was applied for comparison among various groups. The t-test was used for comparison of mean between two samples, Chi-square test was used for comparison of rates. $\mathrm{P}<0.05$ indicated as statistically significant difference.

\section{Results}

Comparison of patients in obesity treatment efficiency, BMI, WHR and glucose metabolism before and after treatments. Two cases in acupuncture-drug group ( 1 in Chinese medicine group and 2 in catgut embedding group) withdrew from the study. Their relevant indexes were no longer examined. As shown in Tables IV and V, obesity treatment efficiency, BMI, WHR, FNS, and HOMA in the three groups were significantly lower after treatment $(\mathrm{P}<0.05)$, and obesity treatment efficiency, BMI, WHR in the acupuncturedrug and catgut embedding groups were all significantly lower than those in the Chinese medicine group $(\mathrm{P}<0.05)$, while the comparison between the acupuncture-drug and catgut embedding groups was of no statistical significance $(\mathrm{P}>0.05)$. FNS and HOMA-IR in the acupuncture-drug group was significantly reduced compared to those in the Chinese medicine and catgut embedding groups $(\mathrm{P}<0.05)$. The comparison between the Chinese medicine group and catgut embedding group was of no statistical significance $(\mathrm{P}>0.05)$. FPG differences in all cases were of no statistical significance (Tables IV and V).

Comparison of the blood lipid of the three groups of patients before treatment. Table VI shows that TG of the three groups of patients after treatment was significantly lower than that of the before treatment $(\mathrm{P}<0.05)$. After treatment, TG of the acupuncture-drug group was significantly lower than that of the Chinese medicine group and catgut embedding group $(\mathrm{P}<0.05)$; HDL of the three groups after treatment was significantly higher than that of the before treatment $(\mathrm{P}<0.05)$. After treatment, HDL of the acupuncture-drug group was significantly higher than that of the Chinese medicine group and catgut embedding group $(\mathrm{P}<0.05)$. The comparison between TG, HDL of Chinese medicine group and those of catgut embedding group had no statistical difference $(\mathrm{P}>0.05)$. TG of the acupuncture-drug group after treatment was significantly lower than that of the before treatment $(\mathrm{P}<0.05)$, the comparison of the before and after treatment of Chinese medicine group and catgut embedding group had no statistical difference (P>0.05). After treatment, TC of the acupuncture-drug group was significantly lower than that of the Chinese medicine group and catgut embedding group $(\mathrm{P}<0.05)$, the comparison 
Table VI. Comparison of TC, TG, LDL and HDL of the three groups of obese patients with PCOS before and after treatment $(\mathrm{mmol} / \mathrm{l}$, mean $\pm \mathrm{SD})$.

\begin{tabular}{lccccc}
\hline Groups/time & Cases & TC & TG & LDL & HDL \\
\hline Acupuncture & & & & & \\
Before treatment & 21 & $4.67 \pm 0.59$ & $2.02 \pm 0.85$ & $2.96 \pm 0.51$ & $1.27 \pm 0.42$ \\
After treatment & 19 & $4.11 \pm 0.52$ & $1.52 \pm 0.49$ & $2.89 \pm 0.49$ & $1.54 \pm 0.33$ \\
Catgut embedding & & & & \\
Before treatment & 21 & $4.48 \pm 0.64$ & $2.19 \pm 0.93$ & $3.11 \pm 0.57$ & $1.18 \pm 0.36$ \\
After treatment & 20 & $4.41 \pm 0.58^{\mathrm{a}}$ & $1.74 \pm 0.58^{\mathrm{a}}$ & $3.02 \pm 0.47$ & $1.23 \pm 0.31^{\mathrm{a}}$ \\
Chinese medicine & & & & \\
Before treatment & 20 & $4.65 \pm 0.77$ & $2.22 \pm 0.79$ & $3.08 \pm 0.55$ & $1.15 \pm 0.41$ \\
After treatment & 18 & $4.51 \pm 0.63^{\mathrm{a}}$ & $1.83 \pm 0.49^{\mathrm{a}}$ & $2.94 \pm 0.51$ & $1.27 \pm 0.30^{\mathrm{a}}$ \\
\hline
\end{tabular}

Comparing with the before treatment, $\mathrm{P}<0.05$; comparing with acupuncture-drug group, ${ }^{\mathrm{a}} \mathrm{P}<0.05$. TC, total cholesterol; TG, triglyceride; LDL, low-density lipoprotein cholesterol; HDL, high-density lipoprotein cholesterol; PCOS, polycystic ovary syndrome.

Table VII. Comparison of the pregnancy result of the three groups of obese patients with PCOS after treatment.

\begin{tabular}{|c|c|c|c|c|c|c|c|}
\hline Groups & Cases & $\begin{array}{l}\text { Treatment } \\
\text { cycle }\end{array}$ & $\begin{array}{c}\text { Periodical } \\
\text { ovulation } \\
\text { rate }(\%)\end{array}$ & $\begin{array}{l}\text { Incidence } \\
\text { rate of } \\
\text { LUF }(\%)\end{array}$ & $\begin{array}{l}\text { Periodical } \\
\text { cancellation } \\
\text { rate }(\%)\end{array}$ & $\begin{array}{l}\text { Incidence } \\
\text { rate of } \\
\text { OHSS }(\%)\end{array}$ & $\begin{array}{c}\text { Pregnancy } \\
\text { rate }(\%)\end{array}$ \\
\hline Acupuncture-drug & 19 & 51 & 74.51 & 7.84 & 5.82 & 1.96 & 35.29 \\
\hline Catgut embedding & 20 & 55 & $54.55^{\mathrm{a}}$ & 10.91 & $16.36^{\mathrm{a}}$ & 1.82 & $12.73^{\mathrm{a}}$ \\
\hline Chinese medicine & 18 & 48 & $52.08^{\mathrm{a}}$ & 8.33 & $14.58^{\mathrm{a}}$ & 2.08 & $14.58^{\mathrm{a}}$ \\
\hline
\end{tabular}

Comparing with acupuncture-drug group, ${ }^{\text {aP }}<0.05$. PCOS, polycystic ovary syndrome; LUF, luteinized unruptured follicle; OHSS, ovarian hyper-stimulation syndrome.

of the Chinese medicine group and catgut embedding group had no statistical difference $(\mathrm{P}>0.05)$. The comparison of LDL of the three group patients before and after treatment had no statistical difference $(\mathrm{P}>0.05)$.

Comparison of the pregnancy result of the three groups after treatment. The periodical ovulation rate and clinical pregnancy of the acupuncture-drug group were significantly higher than those of the Chinese medicine group and catgut embedding group $(\mathrm{P}<0.05)$. The comparison between Chinese medicine group and catgut embedding group had no statistical significance $(\mathrm{P}>0.05)$. The periodical cancellation rate of the acupuncture-drug group was significantly lower than that of the Chinese medicine group and catgut embedding group, which has statistical significance $(\mathrm{P}<0.05)$. The comparison of the Chinese medicine group and catgut embedding group had no statistical significance $(\mathrm{P}>0.05)$. The incidence rate of luteinized unruptured follicle (LUF) and OHSS of the three groups also had no statistical difference $(\mathrm{P}>0.05)$ (Table VII).

Comparison of adverse reactions. During the treatments of patients in the 3 groups, appetite of 24 patients decreased, and they were without the syndromes of nausea, emesis and abdominal pain. Five patients were slightly red and swollen at the site of catgut implantation with white drainage, this was cured by local disinfection to drain out seepage and exposure to TDP light. No anti-inflammation therapy was carried out and no other discomfort was recorded.

\section{Discussion}

PCOS is a syndrome with multiple disease causes and polymorphic clinical features. Its pathophysiological change scope is wide, and it involves unusual changes of nerve, internal secretion, glycometabolism, fat metabolism, protein metabolism and ovarian local regulating factors (8). According to its clinical features, national medicines distribute it to the scope of 'delayed menstrual cycle', 'low menstruation', 'amenorrhea', 'infertility', 'abdominal mass'. Kidney is fundamental to yin and yang of human bodies, and whether the reproductive function of women is normal or not is mainly related to the function of kidney. If kidney gas is abundant, yin and yang are balanced, and then during the menstruation, pregnancy is normal (9). The studies on PCOS have shown that kidney deficiency is the root, and blood stasis is the surface, strengthening kidney and promoting blood circulation is the fundamental method of treating PCOS.

Earlier we identified that there are certain effects of treating endomorphy-type PCOS by using traditional Chinese medicine for nourishing kidney and promoting blood circulation decoction. The kidney-nourishing traditional Chinese medicines are regarded as effect of endocrine hormone. The 
addition of blood circulation activation medicines on the basis of kidney nourishing can improve local blood circulation of ovary, can increase the blood flood volume of ovary, and therefore promote the development of follicles, induce ovulation and promote the formation of corpus luteum, which has been verified in large number of clinical research studies of Chinese and Western medicines (9). At the same time, it has been shown that traditional Chinese medicine treatments alone are dissatisfactory in lowering weight of patients. Therefore, we examined the effect of a kidney-nourishing and blood-promoting decoction in combination with acupoint catgut embedding to treat patients with endomorphy type with PCOS.

The mechanism of acupoint catgut embedding regards catgut as a type of heterogeneous protein, after inserting it into acupoints, its process of softening, decomposition, liquidation and absorption can effectively promote and enhance the nutrition metabolism and stress ability of bodies, vascular permeability and blood circulation can also be improved. It is a type of mixed treatment in combination with multiple treatments and effects, its simulation induction holding time can reach 20 days, which makes up the disadvantages of short period and more visiting times of acupuncture (10).

We selected Renmai, Taiyinjing and Zhongji, Guanyuan, Zhongwan, Shenyu, Xuehai, Sanyinjiao, Fenglong, and Zusanli in Ynagmingjing. Renmai is 'the ocean of Yinmai', Elucidation of Fourteen Channels say Renmai is the foundation of women's health maintenance, the smooth of Renmai could maintain menstruation and fertility functions of women. Spleen is the foundation of acquired functions, spleen meridian, which has the functions of nourishing liver and kidney and regulating thoroughfare and conception vessels, has the meanings of acquired functions nourishing congenital functions. Stomach meridian of foot-yangming belongs to winding spleen stomach, spleen and stomach are the sources of acquired constitution and source of qi-blood. The normal stomach meridian could reconcile qi and blood and make the functions of internal organs normal (5). Apuncture was performed at Guanyuan, with two pinholes in the middle, which could reach two veins of Chong and Rei, and with the qi-blood of three yin of liver-spleen-kidney, which could reach the functions of complementing vigors and adjusting qi-blood. Apuncture at Sanyinjiao could adjust blood circulation, strengthen spleen and remove phlegm, and adjust the three organs of liver, spleen and kidney. In combination with Xuehai and Shenyu, it has the functions of nourishing liver and kidney, adjusting Chong and Ren, and nourishing uterus. Zusanli, Zhongwan and Fenglongxue adjusted spleen and stomach, which make the ascending and descending of spleen and stomach orderly. Endomorphy-type patients with PCOS, deficiency of the kidney and blood stasis were adjusted to reduce weight, the levels of endocrine hormone were adjusted, ovarian functions were improved, ovulation was promoted, menstrual functions were recovered, and therefore the functions of hypothalamus-hypophysis-ovary axis were adjusted.

\section{Acknowledgements}

The present study was supported by the Fund Project from Xuzhou Technology Division (no. KC14SH031).

\section{References}

1. Rotterdam ESHRE/ASRM-Sponsored PCOS Consensus Workshop Group: Revised 2003 consensus on diagnostic criteria and long-term health risks related to polycystic ovary syndrome. Fertil Steril 81: 19-25, 2004.

2. Li M: Diagnosis and treatment of PCOS. Chinese J Obstetrics Gynecol 37: 444-446, 2002 (In Chinese).

3. Goodarzi MO, Dumesic DA, Chazenbalk G and Azziz R: Polycystic ovary syndrome: Etiology, pathogenesis and diagnosis. Nat Rev Endocrinol 7: 219-231, 2011.

4. Hassan A and Gordon CM: Polycystic ovary syndrome update in adolescence. Curr Opin Pediatr 19: 389-397, 2007.

5. Zhen X: Guiding principle of clinical research on new traditional Chinese medicine - Guiding principle of clinical research on new traditional Chinese medicine for the treatment of irregular menstruation. Chinese Medical Science Press, Beijing, p243, 2002.

6. Zhang Y: Traditional Chinese Gynecology (M). China Press of Traditional Chinese Medicine, Beijing, p9, 2002.

7. Weng W and Jiao D: Practical integration of Chinese and Western medicine and bariatrics. Academy Press, Beijing, pp25-27, 1997.

8. Legro RS and Strauss JF III: Molecular progress in infertility: polycystic ovary syndrome. Fertil Steril 78: 569-576, 2002.

9. You Z, Yang Z and Fu L: Discussion of the treatment of kidney deficiency and blood stasis for PCOS. J Hunan College of Traditional Chinese Med 25: 26, 2005.

10. Bogao B and Liugang F: Prediction and experimental research on acupoint catgut embedding for mechanism of fatigue mitigation in rat. J Guangzhou Sport University 33: 95, 2013. 